\title{
Caracterización de genotipos élite de Coffea canephora por su tolerancia a la sequía ${ }^{1}$
}

\section{Characterization of elite Coffea canephora genotypes for its tolerance to drought}

\author{
Ana Odetth Quintana-Escobar², Leobardo Iracheta-Donjuan², Ismael Méndez-López $z^{2}$,Moisés Alonso-Báez²
}

\section{RESUMEN}

El objetivo del presente trabajo fue identificar el grado de tolerancia a sequía en diferentes genotipos élite de Coffea canephora P. var. Robusta. El estudio se realizó del 2013 al 2015 en Tuxtla Chico, Chiapas, México. De cada genotipo (INIFAP P9, INIFAP P4, INIFAP P5, INIFAP P7, INIFAP P8) se utilizaron veinte plantas, excepto para el INIFAP P9 con doce; un grupo con la mitad de plantas de cada genotipo se mantuvo en condiciones de capacidad de campo (CC) y la otra mitad en punto de marchitez permanente (PMP). Al finalizar la etapa de estrés y después del riego de recuperación, se evaluaron variables morfológicas y fisiológicas, tales como parámetros de crecimiento, contenido relativo de agua, acumulación de prolina, índice y densidad estomática e índice de tolerancia a sequía. Los genotipos de $C$. canephora presentaron entre ellos diferente grado de tolerancia al déficit hídrico, pero compartieron entre sí la detención de sus funciones y activación de diferentes rutas para tolerar la sequía, como disminución en el contenido relativo de agua y aumento de prolina durante el periodo de estrés. Al determinar el índice de tolerancia a sequía, el genotipo más tolerante fue el INIFAP P5 de acuerdo con las variables número de hojas retenidas y totales, tasa de crecimiento relativo, número de pares de ramas plagiotrópicas y nudos totales; mientras que el INIFAP P4 se identificó como uno de los más susceptibles de acuerdo con la tasa de crecimiento relativo y el número de nudos totales, en las cuales presentó los valores más bajos.

Palabras clave: déficit hídrico, índice de tolerancia, capacidad de campo.

\begin{abstract}
The aim of the present work was to identify the degree of drought tolerance in different elite genotypes of Coffea canephora P. var. Robusta. The study was conducted from 2013 to 2015 in Tuxtla Chico, Chiapas, Mexico. Twenty plants from each genotype were used (INIFAP P9, INIFAP P4, INIFAP P5, INIFAP P7, INIFAP P8), except for INIFAP P9 with twelve; a group with half of the plants of each genotype was under field capacity (FC) and the other half under permanent wilting point (PWP). Several morphological and physiological variables were considered at the end of the stress stage and after recovery irrigation, such as growth parameters, relative water content, proline accumulation, stomatal index and density and index of drought tolerance. C. canephora genotypes presented different degrees of tolerance to water deficit, and may have activated several pathways to tolerate drought, according to a decrease in relative water content and increase in proline content. Our results suggest that the most tolerant genotype was INIFAP P5 according to the total number of leaves, relative growth rate, and total number of pairs of plagiotropic branches and nodes; while INIFAP P4 was identified as one of the most susceptible according to relative growth rate and total number of nodes.
\end{abstract}

Keywords: water deficit, tolerance index, field capacity.

\footnotetext{
Recibido: 9 de junio, 2016. Aceptado: 26 de julio, 2016. Este trabajo formó parte de un proyecto de investigación realizado en el Instituto Nacional de Investigaciones Forestales, Agrícolas y Pecuarias (INIFAP), México.

2 Instituto Nacional de Investigaciones Forestales, Agrícolas y Pecuarias (INIFAP), Campo Experimental Rosario Izapa, Chiapas, México. Carretera Tapachula-Cacahoatán km 18. Tuxtla Chico Chiapas, México. odetth@gmail.com, iracheta.leobardo@inifap.gob.mx (autor para correspondencia), mendez.ismael@inifap.gob.mx, alonso.moises@inifap.gob.mx
} 


\section{INTRODUCCIÓN}

México es el noveno país productor de café en el mundo (SIAP, 2015). Se cultiva en una extensión de 737 000 ha, donde intervienen 504971 productores y cuyo ingreso por el volumen producido asciende a seis mil millones de pesos. Se cultiva en quince estados, de los cuales Chiapas, Veracruz, Puebla y Oaxaca representan el 93,3\% del total de producción (SHCP, 2014).

El estado de Chiapas concentra más de la tercera parte de la producción con 250661 ha y 180856 productores (AMECAFE, 2012). El 80\% de la producción se localiza en zonas marginales, lo que representa una fuente de ingresos y sustento de productores ejidatarios (AMECAFE, 2012); además, al ser un policultivo, interviene en la conservación de la flora, la fauna y en protección de suelos (Guerrero, 2011), por lo que representa un sistema de producción donde las familias han desarrollado fuertes roles culturales y sociales (Frank et al., 2011).

Las especies Coffea arabica L. (arábica) y $C$. canephora Pierre (Robusta) son las que predominan económicamente en el comercio mundial del café; sin embargo, el café Robusta (C. canephora P.) es más vigoroso y productivo, resistente a plagas y enfermedades, y se desarrolla mejor en climas cálidos que el arábica. La temperatura óptima en que se desarrolla el cultivo oscila entre los $24-26{ }^{\circ} \mathrm{C}$, crece hasta los $800 \mathrm{msnm}$, con abundante precipitación en condiciones de humedad cercanas a la saturación del suelo y una estación seca corta; aunque es sensible a climas extremos (DaMatta y Ramalho, 2006).

Períodos de sequía o cambios en la temporada de lluvias afectan negativamente la fisiología, floración y fructificación del café, lo que provoca disminución de hasta 34\% del rendimiento (Silva et al., 2013).

De acuerdo con algunos modelos de predicción climática, en México se esperan modificaciones en el clima con un aumento de $5{ }^{\circ} \mathrm{C}$ en la temperatura para las próximas décadas (Sáenz-Romero et al., 2012). Para el estado de Chiapas, normalmente afectado por desastres naturales, como huracanes y sequías, se prevé que la frecuencia e intensidad de las mismas aumente. Como consecuencia habrá una disminución de las condiciones adecuadas para el cultivo de café hasta el año 2050 (Schroth et al., 2009).

Es importante contar con genotipos de rendimientos aceptables, capaces de tolerar las condiciones climáticas adversas de los próximos años; tal característica debe ser el primer requisito para un programa de cruzamiento (Cheserek y Gichimu, 2012). Los cultivares con mayor estabilidad de rendimiento en condiciones de sequía y capaces de sobrevivir al estrés, pueden tener mayor valor que aquellos con alto rendimiento seleccionados en ambientes propicios.

Se han realizado estudios de caracterización de materiales tolerantes a condiciones de sequía en campo, en los cuales se ha demostrado que las plantas responden con diversos mecanismos de acuerdo con el genotipo. Las principales respuestas ante el estrés por sequía son: necrosamiento y marchitamiento de hojas, disminución de crecimiento y biomasa, ajuste osmótico, variaciones del estado hídrico, control estomático, mayor profundidad radical y acumulación de solutos; ejemplo de esto último es la prolina, que en ocasiones se utiliza como criterio para la selección de genotipos tolerantes (DaMatta y Ramalho, 2006; Cheserek y Gichimu, 2012).

El índice de tolerancia a sequía se ha utilizado como criterio para seleccionar genotipos por su tolerancia al estrés. Dicho índice consiste en comparar los tratamientos sometidos a estrés de una variable, sobre aquellos en condiciones normales de riego (Castañón et al., 2000; González et al., 2005). Con este índice, se pueden considerar los genotipos con los valores más altos como los más tolerantes; aquellos con los valores más bajos serán los más susceptibles. Por lo anterior, el índice de tolerancia a sequía ha sido ampliamente utilizado en trabajos de tolerancia al estrés.

En México se cuentan con genotipos de $C$. canephora sobresalientes por su rendimiento, dentro de los que figuran INIFAP P9, INIFAP P4, INIFAP P5, INIFAP P7, INIFAP P8, generados por el Instituto Nacional de Investigaciones Forestales, Agrícolas y Pecuarias (INIFAP); sin embargo, se desconoce su grado de tolerancia a sequía, por lo que, al considerar las condiciones climáticas poco favorables que se han suscitado para el cultivo en los últimos años (Schroth et al., 2009), surge la necesidad de conocer el nivel de tolerancia al estrés hídrico por sequía de estos genotipos, así como las respuestas morfofisiológicas involucradas. Por tal motivo, el objetivo fue identificar el grado de tolerancia a sequía en diferentes genotipos élite de Coffea canephora P. var. Robusta. 


\section{MATERIALES Y MÉTODOS}

El trabajo experimental se realizó en los invernaderos del Campo Experimental Rosario Izapa (CERI), del Instituto Nacional de Investigaciones Forestales, Agrícolas y Pecuarias (INIFAP), ubicado en Tuxtla Chico, Chiapas, México. El estudio inició a finales del año 2013 y concluyó en 2014, como parte de un proyecto de investigación finalizado a principios de 2015 .

Se estudiaron cinco genotipos de Coffea canephora P. var. Robusta: INIFAP P9, INIFAP P4, INIFAP P5, INIFAP P7 e INIFAP P8, sobresalientes por tener gran tamaño de semilla, calidad industrial y altos rendimientos. De cada genotipo se utilizaron veinte plantas generadas mediante estacas enraizadas, excepto para el INIFAP P9 con doce plantas, estas se encontraban en un estado fenológico de dos pares de ramas plagiotrópicas y cultivadas en $700 \mathrm{~g}$ de suelo en macetas negras de $35 \times 25 \mathrm{~cm}$ con tres perforaciones para drenado; el suelo fue clasificado por su textura como franco limoso, previamente caracterizado para determinar la capacidad de campo (CC), el punto de marchitez permanente (PMP) y la humedad correspondiente para cada condición de acuerdo con el método de la Olla y membrana de presión (De la Peña, 1977). Se aplicó una fertilización química de N, P, K 17:17:17 (1,2 g/planta) antes del inicio del experimento, de tal modo que no afectara la conductividad eléctrica y salinidad. Las macetas se colocaron en camas de siembra, donde permanecieron durante cuatro meses. La temperatura mínima nocturna y máxima diurna en este ambiente fue de $19{ }^{\circ} \mathrm{C}$ y $32{ }^{\circ} \mathrm{C}$, respectivamente, y humedad relativa promedio de $64,2 \%$.

El total de la población por genotipo se mantuvo por un mes a CC, posteriormente, se dividió en dos grupos: el testigo sin estrés, con diez plantas o repeticiones a CC (seis para el INIFAP P9), cuya humedad edáfica aproximada fue de $33 \%$; el segundo grupo fueron diez plantas (seis para el INIFAP P9), sometidas a condición de estrés por sequía con suspensión de riego durante un mes hasta alcanzar un potencial hídrico del suelo de -2,0 $\mathrm{MPa}$ (-20 bares), correspondiente al PMP cuya humedad edáfica gravimétrica fue de $11 \%$. Se tuvo un total de diez tratamientos, producto de la combinación de los cinco genotipos con dos niveles de humedad del suelo (CC y PMP). La unidad experimental y repetición fue una planta, las cuales se acomodaron de acuerdo con un diseño completamente al azar.
Una vez alcanzado el PMP, esta condición se mantuvo por un lapso de tres días (d), al término del cual se realizó la primera evaluación. Posteriormente, se aplicó un riego de recuperación a CC con riegos constantes cada cinco días. Después del riego de recuperación (ddrr) se evaluó la respuesta a los tres, cinco, diez, quince, veinte, cuarenta y sesenta días en la totalidad de las repeticiones.

Se determinaron la tasa de crecimiento relativo (TCR), de acuerdo con Beadle (1985), con la fórmula $\mathrm{TCR}=(\ln$ altura final $-\ln$ altura inicial $) /$ tiempo $2-$ tiempo 1), dicha altura $(\mathrm{cm})$ se determinó desde el cuello de la planta hasta el meristemo apical; el número de hojas retenidas (NHR), desprendidas (NHD) y totales (NHT); el diámetro del tallo (DT); el número de pares de ramas plagiotrópicas (PRP) y nudos totales (NNT); el número de hojas necrosadas y nivel de necrosis de las mismas, de acuerdo con una gradiente establecida mediante una escala visual arbitraria con cuatro niveles, donde cero correspondió a hojas sin necrosamiento y cuatro a hojas completamente necrosadas (Figura 1); el índice y densidad estomática; el contenido relativo de agua (CRA), de acuerdo con la fórmula descrita por Kramer (1974) como [(peso fresco-peso seco/peso a máxima turgencia-peso seco)*100]; y la prolina según el método de Bates (1973).

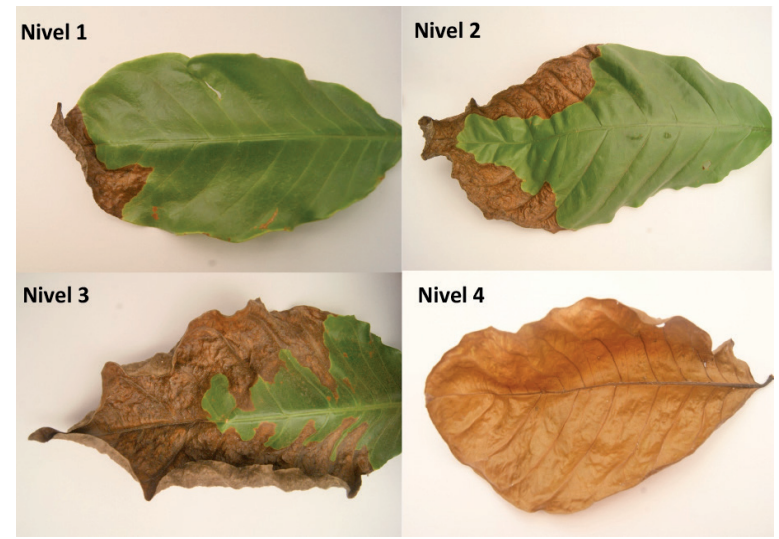

Figura 1. Escala arbitraria para la detección de niveles de necrosamiento en hojas de plantas de Coffea canephora P. var. Robusta, en condiciones de estrés hídrico. Chiapas, México. 2013-2014.

Figure 1. Arbitrary scale for detection of necrosis level in leaves of plants of Coffea canephora P. var. Robusta, under water stress conditions. Chiapas, Mexico. 2013-2014. 
Se determinó la biomasa de vástago y raíz de tres repeticiones por tratamiento a los veinte y sesenta días, y se registró la longitud de la raíz; con los datos biomasa se calculó el índice de calidad de Dickson o ICD (Barajas et al., 2004). El índice de tolerancia a sequía (ITS) se obtuvo con los datos de las variables NHR, TCR, NC, NNT y NHT. El ITS fue el resultado de la diferencia entre los datos de la última y primera evaluación del tratamiento con estrés, dividido entre la diferencia de los datos de la última y primera evaluación del tratamiento sin estrés (López-Peralta, 1984), multiplicado por el valor absoluto del potencial en MPa (2,0 MPa) del PMP por 100.

Se realizó el análisis de varianza y la comparación de medias por el método de Tukey, con nivel de significancia de 0,05 , mediante el paquete estadístico SAS System para Windows versión 9. De igual forma se aplicó un análisis de correlación de Pearson y análisis de componentes principales.

\section{RESULTADOS Y DISCUSIÓN}

Los genotipos presentaron diferencias significativas entre los tratamientos de sequía (PMP) y capacidad de campo (CC). Esta respuesta varió en función de la variable de estudio. Los genotipos mostraron un comportamiento diferente entre ellos, de tal forma que algunos fueron más tolerantes que otros según el índice de tolerancia para la variable utilizada, por lo que esta tolerancia se modificó al utilizar una variable distinta en la fórmula.

En el caso del diámetro de tallo, todos los genotipos tuvieron aumento en grosor después de haber sido sometidos a PMP, con respecto al diámetro observado a los tres días después del riego de recuperación (Cuadro 1). Durante los tres días en estrés, el genotipo INIFAP P5 fue el único que no registró una diferencia significativa del diámetro en las plantas sometidas a PMP, con respecto a las plantas en CC. Sin embargo, transcurridos tres días después del riego de recuperación, los genotipos INIFAP P8, INIFAP P4 e INIFAP P5 fueron capaces de restablecer su crecimiento en grosor de las plantas estresadas, pues no se observaron diferencias significativas con respecto al testigo en CC. Esta capacidad de recuperar el crecimiento del tallo en grosor, podría estar asociada a aquellos genotipos que presentan una
Cuadro 1. Diámetro del tallo de cinco genotipos de Coffea canephora P. var. Robusta a los tres días en PMP y a los tres días después del riego de recuperación (ddrr) en condiciones de capacidad de campo (CC) y punto de marchitez permanente (PMP). Chiapas, México. 2013-2014.

Table 1. Stem diameter for five genotypes of Coffea canephora P. var. Robusta, at three days at the permanent whilting point (PMP) and three days after the recovery irrigation (ddrr) in field capacity (FC) and permanent wilting point (PWP) conditions. Chiapas, Mexico. 2013-2014.

\begin{tabular}{lccc}
\hline Genotipo & Humedad & \multicolumn{2}{c}{ Diámetro (cm)* } \\
\cline { 3 - 4 } & & 3 días PMP & 3 días ddrr \\
\hline INIFAP P7 & CC & $6,1 \mathrm{a}$ & $6,3 \mathrm{a}$ \\
& PMP & $5,1 \mathrm{~b}$ & $5,6 \mathrm{~b}$ \\
& C.V.** & 5,5 & 3,9 \\
\hline INIFAP P8 & CC & $6,5 \mathrm{a}$ & $6,2 \mathrm{a}$ \\
& PMP & $5,5 \mathrm{~b}$ & $6,9 \mathrm{a}$ \\
& C.V. & 4,0 & 5,6 \\
\hline INIFAP P9 & CC & $6,4 \mathrm{a}$ & $6,9 \mathrm{a}$ \\
& PMP & $5,5 \mathrm{~b}$ & $6,3 \mathrm{~b}$ \\
& C.V. & 3,4 & 2,9 \\
\hline INIFAP P4 & CC & $6,4 \mathrm{a}$ & $5,3 \mathrm{a}$ \\
& PMP & $5,6 \mathrm{~b}$ & $5,8 \mathrm{a}$ \\
& C.V. & 4,1 & 6,3 \\
\hline INIFAP P5 & CC & $5,2 \mathrm{a}$ & $5,2 \mathrm{a}$ \\
& PMP & $5,0 \mathrm{a}$ & $5,2 \mathrm{a}$ \\
& C.V. & 4,0 & 5,3 \\
\hline
\end{tabular}

* Medias con diferente letra por columna por genotipo, son significativamente diferentes (Tukey $\mathrm{p} \leq 0,05$ ) / Means followed by different letters in each column are significantly different (Tukey test $\mathrm{p} \leq 0.05$ )

** C.V.: coeficiente de variación / C.V.: variation coefficient.

mayor velocidad de crecimiento, aun en condiciones de estrés, comparados con los genotipos INIFAP P9 e INIFAP P7.

La tasa de crecimiento relativo (TCR) fue menor en las plantas sometidas a PMP, en comparación con aquellas en CC para cada genotipo a los tres días en PMP, con excepción del genotipo INIFAP P5, donde la actividad de crecimiento del meristemo apical se mantuvo sin cambio entre ambas condiciones (Figura 2). 

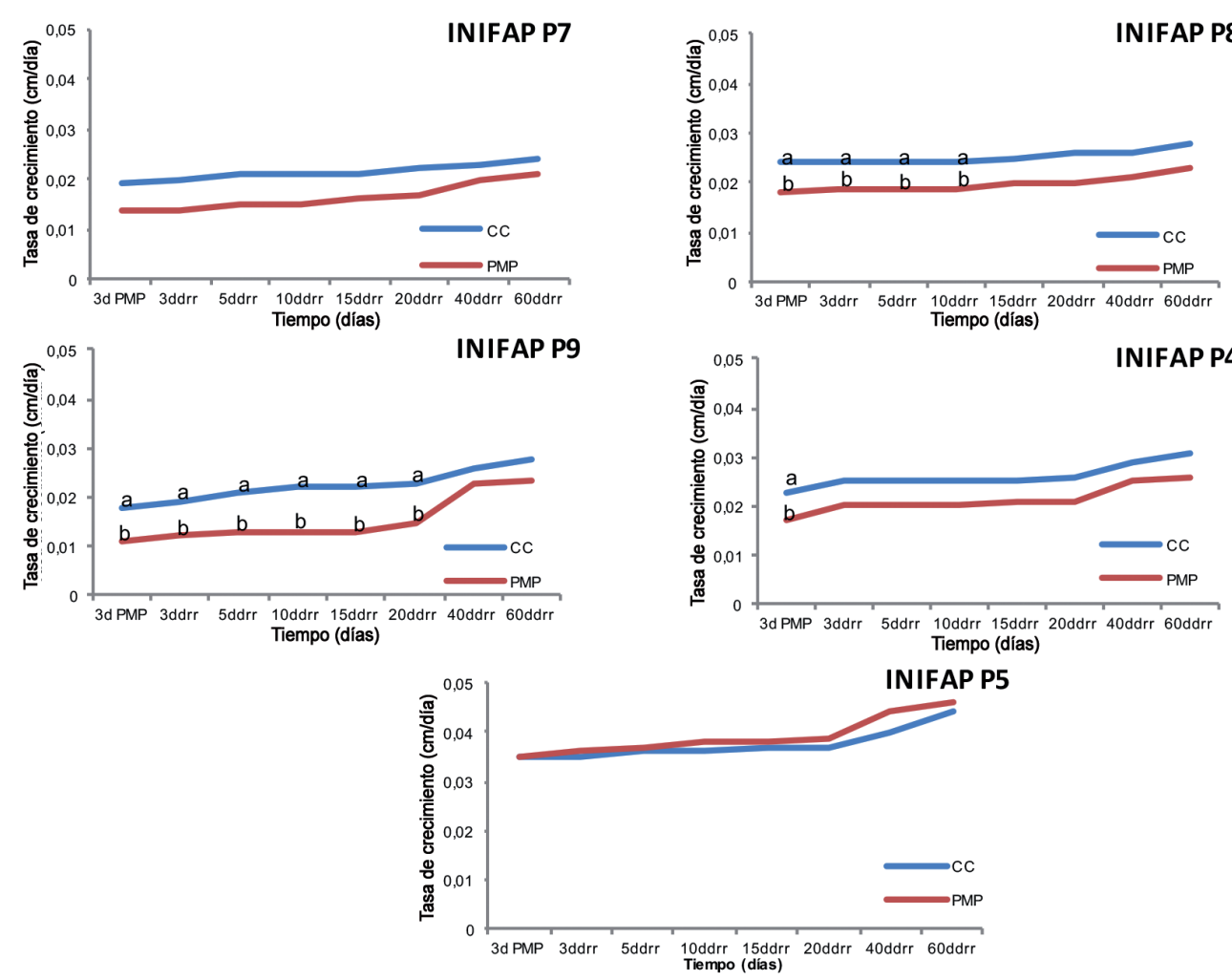

Figura 2. Tasa de crecimiento relativo ( $\mathrm{cm} /$ día) de cinco genotipos de Coffea canephora P. var. Robusta en condiciones de capacidad de campo (CC) y punto de marchitez permanente (PMP). Promedios con letras distintas por cada fecha son significativamente diferentes (Tukey $\mathrm{p} \leq 0,05$ ). Chiapas, México. 2013-2014.

ddrr: días después del riego de recuperación.

Figure 2. Relative growth rate (cm/day) of five genotypes of Coffea canephora P. var. Robusta, in field capacity (FC) and permanent wilting point (PWP) conditions. Averages with different letters for each date are significantly different (Tukey test $\mathrm{p} \leq 0.05$ ). Chiapas, Mexico. 2013-2014.

ddrr: days after recovery irrigation.

Lo anterior indica una respuesta diferencial del genotipo a la condición de sequía.

En los genotipos INIFAP P8, INIFAP P9 e INIFAP P4, la TCR de las plantas sometidas a estrés fue significativamente diferente de aquellas a CC para cada genotipo a los tres días en PMP (Figura 2); sin embargo, a los tres días después del riego de recuperación, solo las plantas del genotipo INIFAP P4 en condiciones de PMP fueron capaces de igualar significativamente la TCR con respecto a las plantas a CC, mientras que el INIFAP P8 y el INIFAP P9 se recuperaron pasados diez y veinte días después de aplicado el riego de recuperación. El retraso en la recuperación de la tasa de crecimiento de los genotipos INIFAP P8 e INIFAP P9, denotó una mayor sensibilidad de ambos genotipos a la condición de estrés por sequía. Villar et al. (2004) mencionaron al respecto, que la limitación de humedad en las plantas propicia una tasa de crecimiento más lenta, ello debido a que la planta en estrés destina su energía a la síntesis de compuestos defensivos (con un costo metabólico) en lugar de invertir su energía en crecimiento, mantenimiento y reproducción. Por otro lado, si bien el genotipo INIFAP P7 presentó algunas diferencias numéricas entre la condición de déficit hídrico y riego constante, estas se mantuvieron 
en el umbral en el cual no se detectaron diferencias significativas para la TCR entre las plantas sometidas a CC y PMP, aún en el periodo de estrés.

Las plantas del genotipo INIFAP P5 sometidas a PMP, toleraron mejor la condición de estrés por sequía, ya que no mostraron diferencias estadísticas desde la etapa de estrés hasta el término del experimento; incluso fue posible observar que, desde el riego de recuperación, las plantas que habían estado en PMP tuvieron un crecimiento ligeramente mayor que aquellas que estuvieron todo el tiempo a CC.
Si bien, las plantas de todos los genotipos presentaron disminución del número de nudos totales (NNT) en la condición de sequía (Figura 3), solo en los genotipos INIFAP P7 e INIFAP P5, dicha disminución fue significativa; además, ambos materiales mantuvieron esta diferencia aún quince y veinte días después de aplicado el riego de recuperación, respectivamente. Lo anterior contradice a lo observado en la tasa de crecimiento relativo, en la cual, como ya se mencionó, ambos genotipos se mantuvieron sin cambio en ambas condiciones. Esto
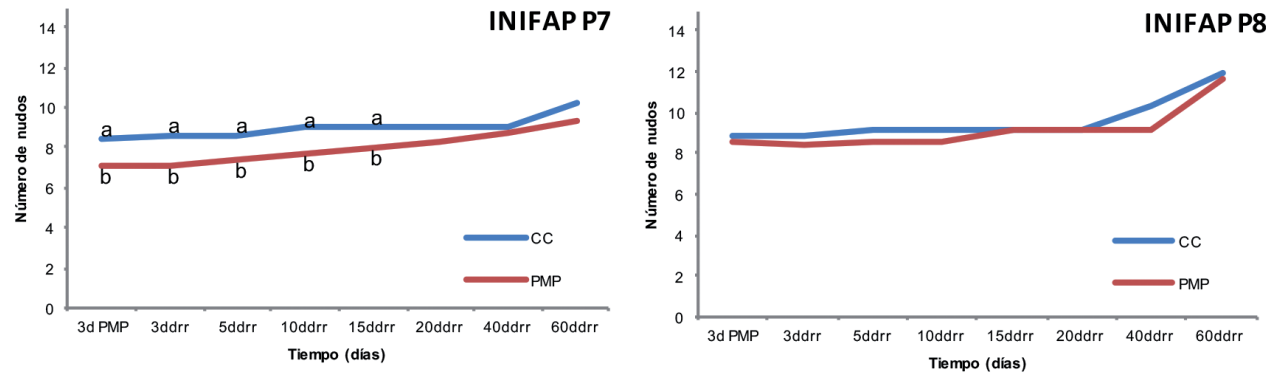

INIFAP P9
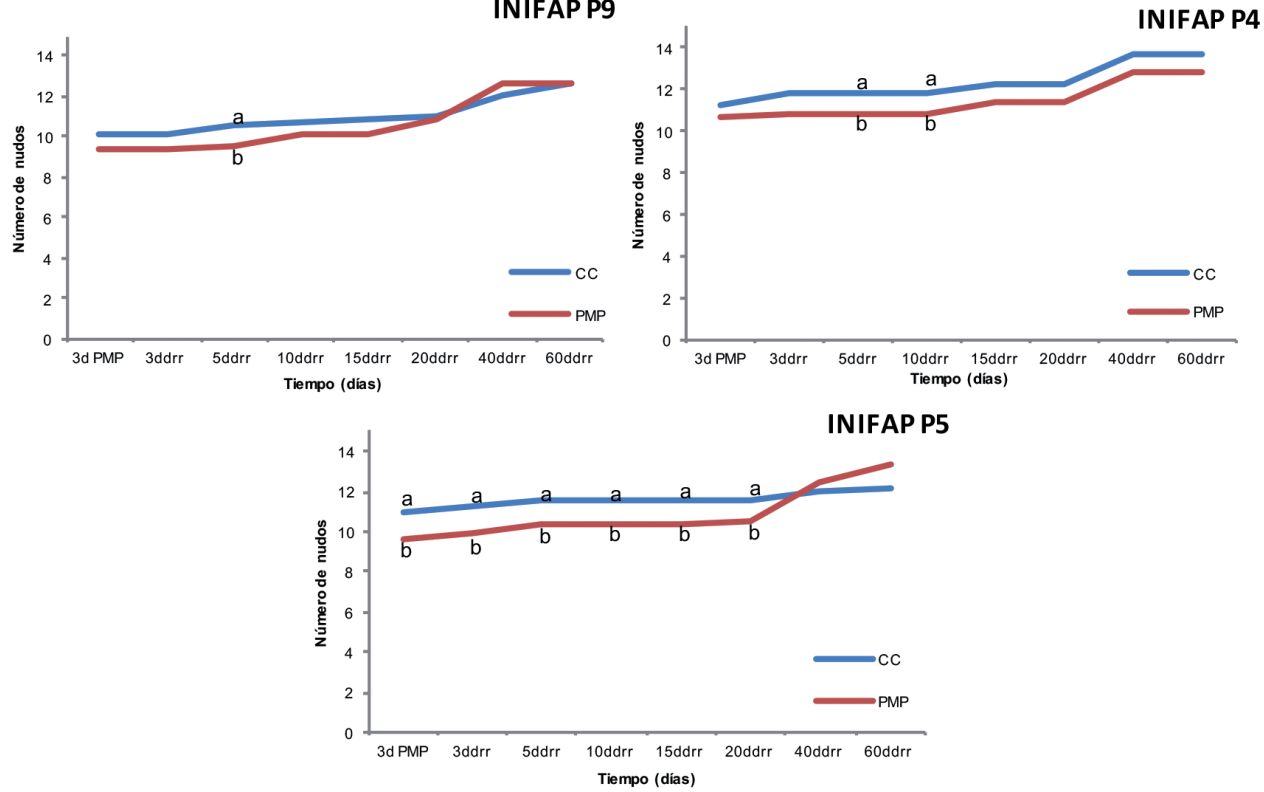

Figura 3. Número de nudos totales de cinco genotipos de Coffea canephora P. var. Robusta en condiciones de capacidad de campo (CC) y punto de marchitez permanente (PMP). Promedios con letras distintas por cada fecha son significativamente diferentes (Tukey $\mathrm{p} \leq 0,05$ ). Chiapas, México. 2013-2014.

ddrr: días después del riego de recuperación.

Figure 3. Total number of nodes in five genotypes of Coffea canephora $\mathrm{P}$. var. Robusta, in field capacity (FC) and permanent wilting point (PWP) conditions. Averages with different letters for each date are significantly different (Tukey test $\mathrm{p} \leq 0.05$ ). Chiapas, Mexico. 2013-2014. ddrr: days after recovery irrigation. 
indica que las plantas mantuvieron el crecimiento en las dos condiciones hídricas, sin que ello signifique la aparición de nuevos nudos, es decir, que el crecimiento registrado solo se debe al alargamiento de los entrenudos ya existentes y no al rebrote. Paula (2004) señala que dicha capacidad de rebrote de plantas estresadas, por defoliación masiva, depende de la acumulación de carbohidratos no estructurales de reserva (almidón, sacarosa, fructosa y glucosa); consigna que las diferencias de rebrote entre especies de brezo (Erica sp.) fue debida al diferente grado de acumulación de carbohidratos de reserva.

Por otro lado, únicamente los genotipos INIFAP P8 e INIFAP P9 mantuvieron valores estadísticamente similares para el NNT entre las plantas sometidas a PMP y a CC, durante y después del periodo de estrés. Las plantas sometidas a estrés por sequía lograron igualar estadísticamente el número de nudos totales de las plantas a CC a partir de los diez días después del riego de recuperación en adelante, dependiendo del genotipo. Incluso, las plantas del genotipo INIFAP P5 que fueron sometidas a estrés, superaron numéricamente el total de nudos de las plantas a CC a partir de los cuarenta días. Esta mayor recuperación del genotipo INIFAP P5, podría estar asociada a una mayor capacidad para inducir rebrotes aun en condiciones hídricas adversas, tal como lo señalaron Villar et al. (2004), quienes mencionan que la mayoría de las plantas sometidas a condiciones adversas disminuyen el crecimiento o desarrollo, pero no descarta la existencia de plantas con mayor tolerancia, capaces de tener altas tasas de crecimiento y continuar con su desarrollo, aún durante periodos de estrés.

En el caso de contenido relativo de agua (CRA), las plantas de la mayoría de los genotipos presentaron disminución entre ambas condiciones durante los tres días en estrés ( 3 d PMP); sin embargo, solo en los genotipos INIFAP P8 e INIFAP P4, estas diferencias entre la condición de CC y PMP fue significativa (Figura 4). Lo anterior implica que en todos los materiales se presentó pérdida de CRA, pero esta varió de acuerdo con el genotipo, puesto que el INIFAP P8 e INIFAP P4 presentaron pérdidas de 28 a $31 \%$, respectivamente, comparados con el resto de los genotipos cuyas pérdidas oscilaron entre 9 y $19 \%$, lo cual podría estar asociado con cambios en las relaciones hídricas de los tejidos. Al respecto, se ha reportado que el porcentaje de CRA de las plantas de $C$. dodecandra, P. piscipula y L. leucocephala sometidas a estrés, varía según la especie y disminuye proporcionalmente con la reducción del potencial del suelo, en comparación con aquellas sin estrés (LunaFlores et al., 2012).

Este contenido relativo de agua aumentó ligeramente conforme se aplicó riego constante. Sin embargo, para los genotipos INIFAP P9, INIFAP P4 e INIFAP P5, a los diez días ddrr, a pesar de estar en riego constante, se apreció un descenso en el CRA de las plantas. En este sentido, Lamz y González (2013) aseveraron que una disminución del potencial hídrico del suelo, invariablemente se reflejará en el estado hídrico de la planta y por consiguiente, en el CRA, ya que los tejidos tenderán a perder agua con la finalidad de mantener un potencial hídrico más negativo que el del suelo, y así asegurar el movimiento continuo del agua entre el suelo y la planta.

Con los resultados anteriores, fue posible confirmar que la condición hídrica del suelo propició un cambio variable en las relaciones hídricas entre el continuo suelo-planta-atmósfera, el cual varió de acuerdo con el genotipo de café; dicho cambio se vio reflejado en el CRA de cada genotipo.

Los cambios observados en el CRA en cada genotipo utilizado en la presente investigación, repercutieron de forma diferente en la respuesta de otras variables durante la condición de estrés. Sin embargo, después del riego de recuperación, todos los genotipos fueron capaces de igualar el contenido relativo de agua entre las dos condiciones hídricas, sin que ello significara la recuperación al mismo tiempo de otras variables de estudio.

A los tres días en PMP, todos los genotipos mostraron diferencias significativas en el contenido de prolina entre la condición de CC y PMP. Las plantas en tratamiento de sequía (PMP) tuvieron valores de prolina notablemente más elevados (Figura 5). La acumulación de prolina se ha relacionado con múltiples eventos, como son degradación de las proteínas solubles en las hojas, pérdida de clorofila, reducción de actividad mitocondrial y translocación de compuestos nitrogenados de las hojas senescentes (Llano y Alcaraz, 2012). De igual forma, se ha demostrado que la prolina juega un papel importante ante el estrés por sequía; por lo que, se relaciona con la disminución del potencial hídrico y el contenido relativo de agua.

Tres días después del riego de recuperación, las plantas en PMP de los genotipos INIFAP P7 e INIFAP 

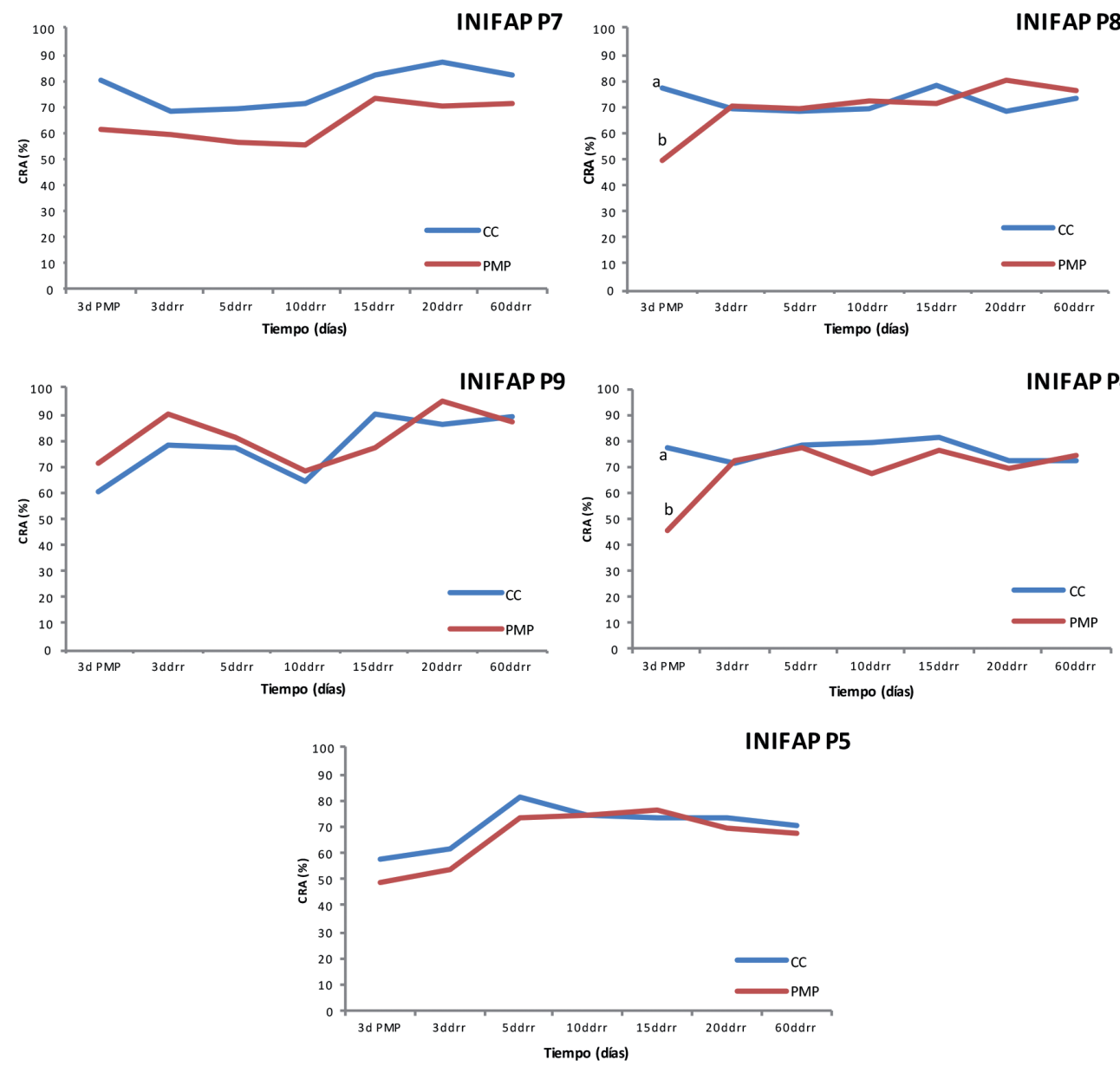

Figura 4. Porcentaje de contenido relativo de agua (CRA) de cinco genotipos de Coffea canephora P. var. Robusta en condiciones de capacidad de campo (CC) y punto de marchitez permanente (PMP). Promedios con letras distintas por fecha son significativamente diferentes (Tukey $\mathrm{p} \leq 0,05$ ). Chiapas, México. 2013-2014.

ddrr: días después del riego de recuperación.

Figure 4. Percentage of relative water content (CRA) of five genotypes of Coffea canephora P. var. Robusta, in field capacity (FC) and permanent wilting point (PWP conditions. Averages with different letters for each date are significantly different (Tukey test $\mathrm{p} \leq 0.05$ ). Chiapas, Mexico. 2013-2014. ddrr: days after recovery irrigation.

P8 igualaron temporalmente el contenido de prolina de las plantas sometidas a CC; sin embargo, esta condición no se mantuvo en la evaluación posterior. Lo contrario sucedió en el INIFAP P4, donde la igualdad entre las plantas en condiciones de PMP y CC fue permanente a partir de los tres días ddrr. Mientras que, entre los veinte y cuarenta días, todas las plantas que fueron sometidas a condiciones de PMP, igualaron los valores de concentración de prolina de las plantas sometidas a CC, excepto el INIFAP P8, en el que se observaron diferencias significativas entre ambas condiciones hasta los sesenta días (Figura 5).

El hecho de que aún haya niveles elevados de prolina después del riego de recuperación, fue explicado por Avendaño et al. (2005), quienes en el caso de plantas de maíz sometidas a sequía, consideraron 

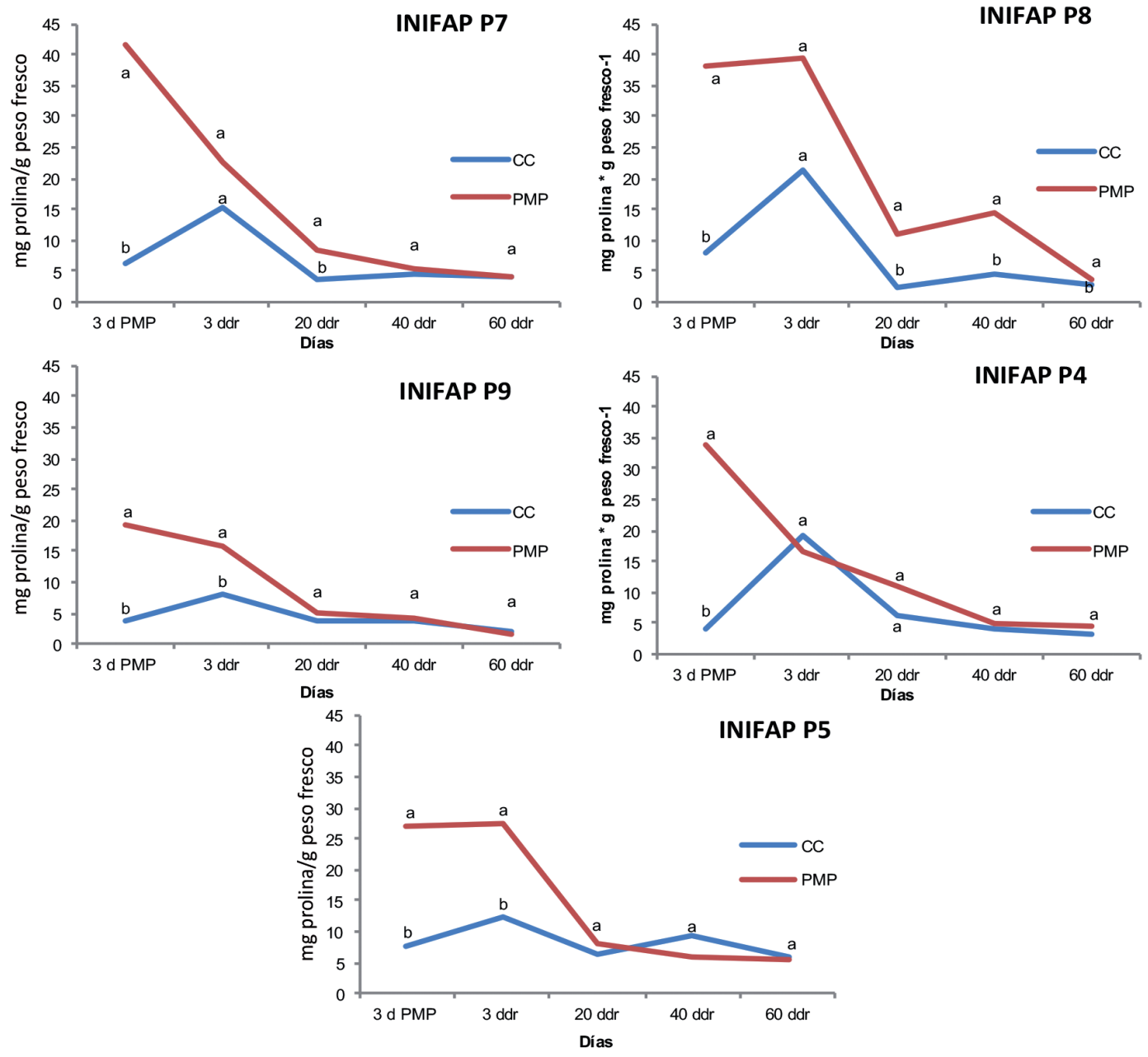

Figura 5. Contenido de prolina en cinco genotipos de Coffea canephora P. var. Robusta, bajo condiciones de capacidad de campo (CC) y punto de marchitez permanente (PMP). Promedios con letras distintas por cada fecha son significativamente diferentes (Tukey p $\leq 0,05)$. Chiapas, México. 2013-2014.

ddrr: días después del riego de recuperación.

Figure 5. Proline content in five genotypes of Coffea canephora P.var. Robusta, under field capacity (FC) and permanent wilting point (PWP) conditions. Averages with different letters for each date are significantly different (Tukey test $\mathrm{p} \leq 0.05$ ). Chiapas, Mexico. 2013-2014.

ddrr: days after recovery irrigation.

que los altos niveles de prolina registrados en algunos genotipos después del riego de recuperación, se debió a que el mecanismo de síntesis de prolina continuó activo después del riego, por lo que las plantas continuaron con la síntesis de este aminoácido para tener una mayor recuperación.
Para el porcentaje de hojas retenidas, se observó que el genotipo INIFAP P7 fue el único que retuvo el 100\% de las hojas hasta transcurridos cinco días después del riego de recuperación (ocho días después de llegar a PMP). Mientras que el INIFAP P9 solo retuvo el $100 \%$ hasta los tres días después del riego 
de recuperación (Figura 6). El resto de los genotipos disminuyeron el porcentaje de hojas durante los tres días en PMP. De los diez a los veinte días después del riego de recuperación, la mayoría de los genotipos disminuyeron las hojas entre 76 y $84 \%$. Por su parte, el genotipo INIFAP P8 fue el más afectado a lo largo de todas las evaluaciones, con porcentajes que cambiaron de 91 a $62 \%$.

La reducción del número de hojas se atribuye a un descenso en la expansión celular, producto de la disminución de la turgencia y contenido de agua en la planta, esto es considerado como un mecanismo de adaptación al estrés por sequía, debido a que favorece la disminución de la tasa transpiratoria (Marraccini et al., 2012), y de esta forma se contrarresta el déficit hídrico de las hojas que quedan retenidas en la planta (Mamani-Rojas y François-Ledent, 2014).

Los genotipos INIFAP P9, INIFAP P4 e INIFAP P5 presentaron una recuperación de hojas a partir de los cuarenta días después del riego de recuperación mediante la formación de nuevas hojas. Es importante señalar que el genotipo INIFAP P7 continuó con una pérdida gradual de hojas, aunque no al grado del genotipo INIFAP P8.

En la Figura 7 se muestran las imágenes del sistema radical de todos los genotipos, tanto en condiciones de PMP como en CC. En ella se aprecia que las plantas sometidas a PMP de los genotipos INIFAP P8, INIFAP P9 e INIFAP P5 indujeron una raíz principal con mayor elongación hacia el meristemo apical (zona de crecimiento), mientras que en los genotipos restantes se dio un crecimiento más denso de raíces secundarias en la zona de ramificación. Lo anterior demuestra que los genotipos utilizaron estrategias diferentes para hacer frente a la condición de estrés. Esta característica de mayor crecimiento del sistema radical podría influir en la tasa relativa de crecimiento de tales genotipos.

En todos los genotipos se observó disminución en la acumulación de biomasa seca en la condición de estrés hídrico (Figura 8); no obstante, el genotipo más afectado en la acumulación seca en vástago y raíz fue el INIFAP P8, con una diferencia de 8 y 16 g, respectivamente, en plantas sometidas a CC. Mientras

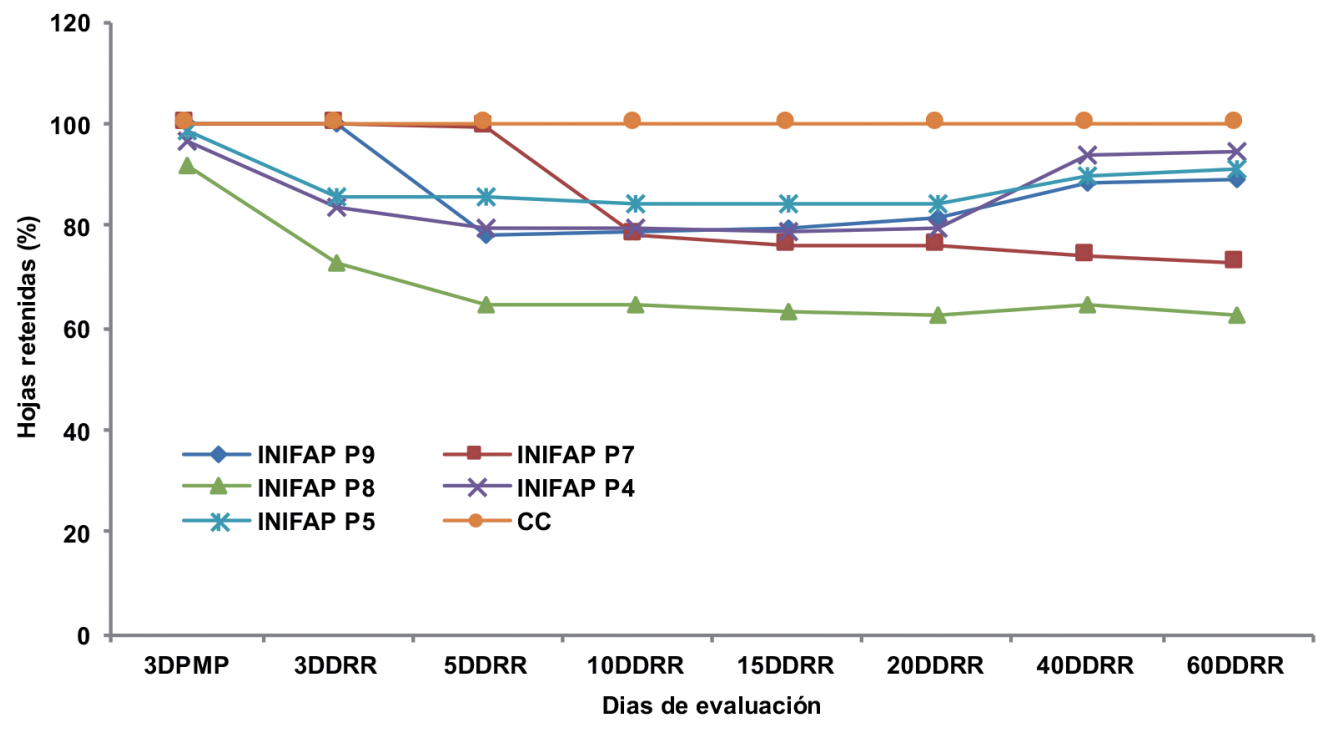

Figura 6. Porcentaje de hojas retenidas de cinco genotipos de Coffea canephora P. var. Robusta bajo condiciones de punto de marchitez permanente (PMP). Chiapas, México. 2013-2014. CC: porcentaje de hojas retenidas para los cinco genotipos en capacidad de campo. DDRR: días después del riego de recuperación.

Figure 6. Percentage of retained leaves of five genotypes of Coffea canephora P. var. Robusta, under permanent wilting point (PMP) conditions. Chiapas, Mexico. 2013-2014.

CC: percentage of retained leaves of five genotypes under field capacity. DDRR: days after recovery irrigation. 


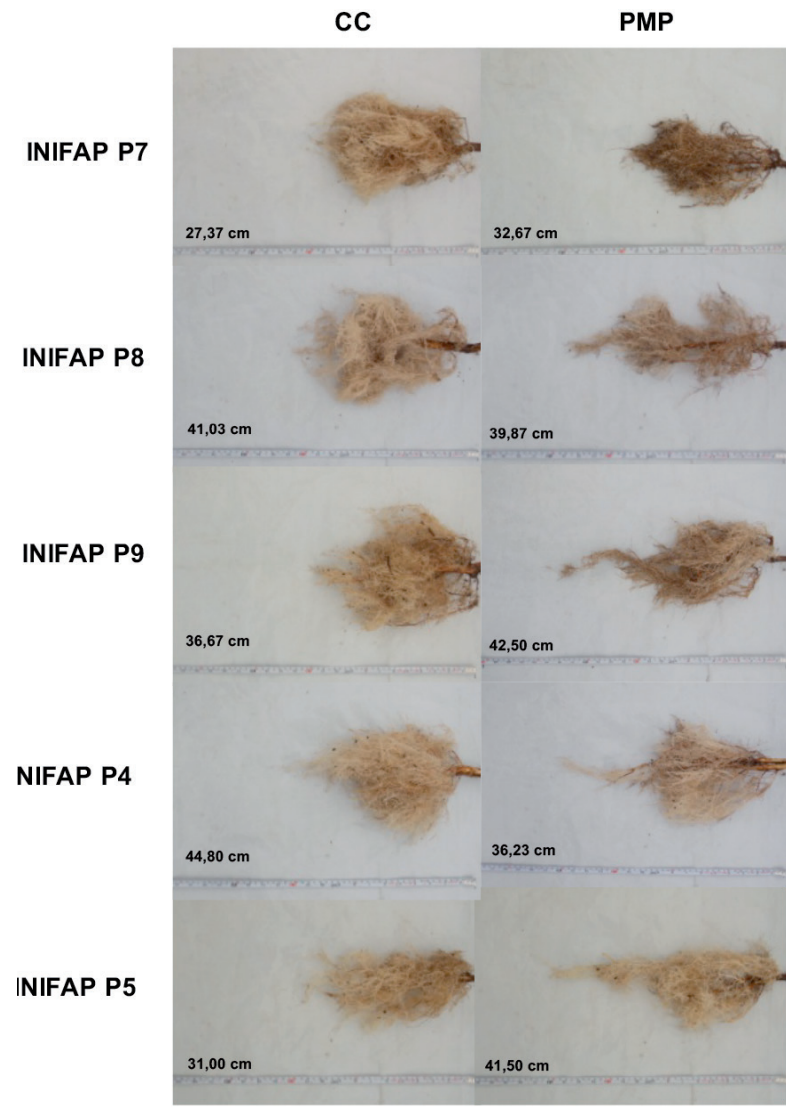

Figura 7. Longitud (cm) del sistema radical de cinco genotipos de $C$. canephora P. var. Robusta en condiciones de capacidad de campo (CC) y punto de marchitez permanente (PMP) a los sesenta días después del riego de recuperación. Chiapas, México. 2013-2014.

Figure 7. Length $(\mathrm{cm})$ of root system of five genotypes of $C$. canephora $\mathrm{P}$. var. Robusta, in field capacity (FC) and permanent wilting point (PWP) conditions at sixty days after the recovery irrigation. Chiapas, Mexico. 2013-2014.

los genotipos menos afectados correspondieron al INIFAP P5 e INIFAP P7. A los sesenta días después del riego de recuperación, en el tratamiento sometido a estrés del genotipo INIFAP P5 se registró mayor contenido de biomasa que en el testigo. Resultados similares se observaron en Tagetes minuta, donde las plantas estresadas tuvieron mayor biomasa que las no estresadas (Mohamed et al., 2000). Este efecto puede estar asociado al contenido de solutos acumulados en las plantas como respuesta ante el estrés, tales como proteínas, minerales y ácidos grasos.

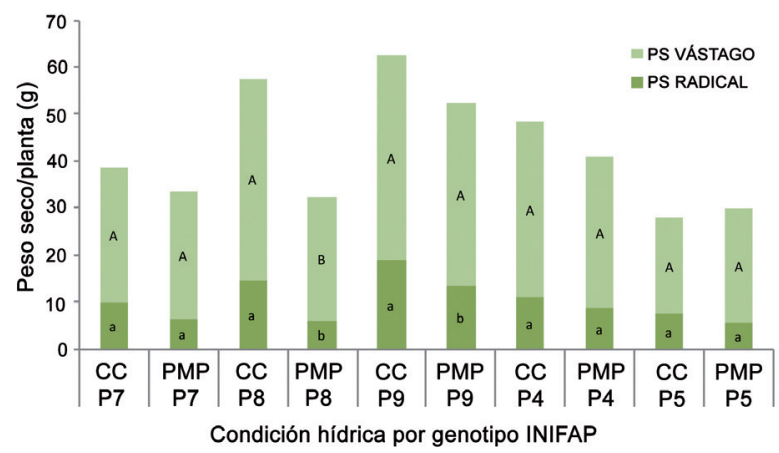

Figura 8. Peso seco (PS) de vástago y raíz de cinco genotipos de C. canephora P. var. Robusta en condiciones de capacidad de campo (CC), y punto de marchitez permanente (PMP) a los sesenta días después del riego de recuperación. Promedios con letras distintas entre condición de humedad por genotipo indican diferencia significativa (Tukey $\mathrm{p} \leq 0,05$ ). Chiapas, México. 2013-2014.

Figure 8. Stem and root dry weigth (PS) of five genotypes of C. canephora $\mathrm{P}$. var. Robusta, in field capacity (FC) and permanent wilting point (PWP) conditions at sixty days after the recovery irrigation. Averages with different letters between moisture conditions for genotype are significantly different (Tukey test $\mathrm{p} \leq 0.05)$. Chiapas, Mexico. 2013-2014.

Los genotipos presentaron diferente nivel de tolerancia al necrosamiento de las hojas. Para el caso del nivel uno de necrosamiento (solo punta de hojas necrosadas) el genotipo INIFAP P9 presentó mayor incidencia comparado con los otros genotipos para este mismo nivel; aunque el porcentaje fue bajo, pues no superó el $10 \%$. Los niveles dos y tres de necrosamiento de hoja se mantuvieron relativamente bajos (menor a 5\%) en todos los genotipos. Sin embargo, para el nivel cuatro de necrosamiento (hoja totalmente necrosada), fue en el que mostró con mayor frecuencia en todos los genotipos. No obstante, el genotipo INIFAP P8 registró la mayor afectación por este tipo de daño en el 46,3\% de sus hojas, seguido por el INIFAP P7, INIFAP P4, INIFAP P9 y finalmente por el INIFAP P5 (con 15,6\%). Tal necrosamiento de las hojas fue una de las manifestaciones más visibles del marchitamiento provocado por el estrés, lo cual puede producir daños irreversibles en las plantas. $\mathrm{Al}$ respecto, varios autores consideran que tal necrosamiento trae consigo la pérdida de turgencia celular y un periodo prolongado con estomas cerrados, además del sobrecalentamiento de las hojas debido a la ausencia de la transpiración (Martínez et al., 1994). 
En cuanto al índice de calidad de Dickson (ICD) no se registraron diferencias significativas entre las condiciones hídricas a CC y PMP para la mayoría de los genotipos, excepto en el INIFAP P8 (ICD=4,109 en CC y 2,199 en PMP), el cual registró disminución en la calidad de planta de forma significativa; lo anterior implica que el aspecto de la planta es modificado por el estrés y que, la pérdida de hojas, la disminución de TCR y el crecimiento de la raíz, podrián contribuir a que el genotipo INIFAP P8 sea uno de los materiales más susceptibles a la sequía, mientras que el INIFAP P7 y sobre todo el genotipo INIFAP P5 fueron más tolerantes. Resultados similares fueron reportados en plantas de Eucalyptus grandis, donde fue posible detectar tratamientos (a base de sustratos y riegos adecuados) que mejoraron el ICD, mientras que otros propiciaron una respuesta contraria, la cual fue asociada a un desbalance en el desarrollo de la parte área y radical (Castillo et al., 2008).

Por otra parte, Navarro-Sandoval et al. (2013) señalan que en plantas de vivero de Avies religiosa las heladas no influyeron en el ICD, tanto en plantas fertilizadas como en las que no lo fueron; esto fue asociado a que los daños presentes en el ápice del brote fueron leves. Lo anterior estuvo relacionado con lo observado en este trabajo para la mayor parte de los genotipos, no así para el INIFAP P8, donde la disminución de la TCR denotó una afectación del crecimiento en el ápice de brote en condiciones de estrés.

No se encontraron diferencias significativas para los genotipos en el índice estomático (IE) de las plantas, entre el tratamiento sometido a estrés y el testigo a CC (Cuadro 2). Sin embargo, en la mayoría de los genotipos, el tratamiento bajo estrés presentó mayor índice estomático numéricamente. De igual manera, otros autores (Salas et al., 2001), encontraron un efecto del estrés sobre las variaciones en el índice estomático (IE), donde los valores de IE fueron mayores en los tratamientos sometidos a estrés. Tales variaciones están relacionadas con la distribución de los estomas en las hojas.

No se registró diferencia entre ambas condiciones hídricas para la densidad estomática (DE), ya que esta mantuvo valores similares entre los dos tratamientos para la mayoría de los genotipos. En el caso del INIFAP P5, se observó que hubo mayor densidad en la condición sin estrés (Figura 9), lo cual se relaciona con los cambios observados en el cultivo de tomate (Salas et al., 2001) en respuesta al estrés, tal como
Cuadro 2. Índice y densidad estomática en hojas de plantas de cinco genotipos de $C$. canephora sometidos a dos condiciones hídricas (sin estrés y con estrés hídrico). Chiapas, México. 2013-2014.

Table 2. Index and stomatic density in plant leaves of five genotypes of $C$. canephora under to two water conditions (without and with water stress). Chiapas, Mexico. 2013-2014.

\begin{tabular}{lccc}
\hline Genotipo & Tratamiento & $\begin{array}{c}\text { Índice } \\
\text { estomático }\end{array}$ & $\begin{array}{c}\text { Densidad } \\
\text { estomática }\end{array}$ \\
\hline INIFAP P7 & Sin estrés & $18,9 \mathrm{a}^{*}$ & $260,3 \mathrm{a}$ \\
& Con estrés & $21,4 \mathrm{a}$ & $266,6 \mathrm{a}$ \\
& C.V.** & 9,2 & 10,7 \\
\hline INIFAP P8 & Sin estrés & $19,1 \mathrm{a}$ & $247,6 \mathrm{a}$ \\
& Con estrés & $18,6 \mathrm{a}$ & $234,9 \mathrm{a}$ \\
& C.V. & 7,4 & 10,0 \\
\hline INIFAP P9 & Sin estrés & $19,3 \mathrm{a}$ & $237,0 \mathrm{a}$ \\
& Con estrés & $21,5 \mathrm{a}$ & $281,5 \mathrm{a}$ \\
& C.V. & 10,4 & 14,5 \\
\hline INIFAP P4 & Sin estrés & $19,8 \mathrm{a}$ & $241,2 \mathrm{a}$ \\
& Con estrés & $23,3 \mathrm{a}$ & $298,4 \mathrm{a}$ \\
& C.V. & 8,2 & 9,9 \\
\hline INIFAP P5 & Sin estrés & $20,2 \mathrm{a}$ & $234,9 \mathrm{a}$ \\
& Con estrés & $17,9 \mathrm{a}$ & $184,1 \mathrm{~b}$ \\
& C.V. & 7,7 & 8,6 \\
\hline
\end{tabular}

*Medias con diferente letra por columna por genotipo, son significativamente diferentes (Tukey $\mathrm{p} \leq 0,05$ ) / Means with different letters in columns by genotype are significantly different (Tukey test $\mathrm{p} \leq 0.05)$.

** C.V.: coeficiente de variación / C.V.: variation coefficient.

disminución del número de estomas y aumento de tamaño de células epidérmicas. Algunos autores (Salas et al., 2001; Toral et al., 2010), han consignado que esta disminución en la densidad estomática en la condición de estrés podría estar relacionada con el aumento de la resistencia estomática, la cual evita el exceso de transpiración y pérdida de agua, lo que da lugar a una mejor adaptación del cultivo a las condiciones de estrés hídrico.

En muchos de los resultados anteriormente expuestos se destaca el genotipo INIFAP P5 como uno de los menos afectados por la condición de estrés. Tal respuesta se confirmó mediante el índice de tolerancia a sequía, en el cual, el genotipo INIFAP P5 fue el 

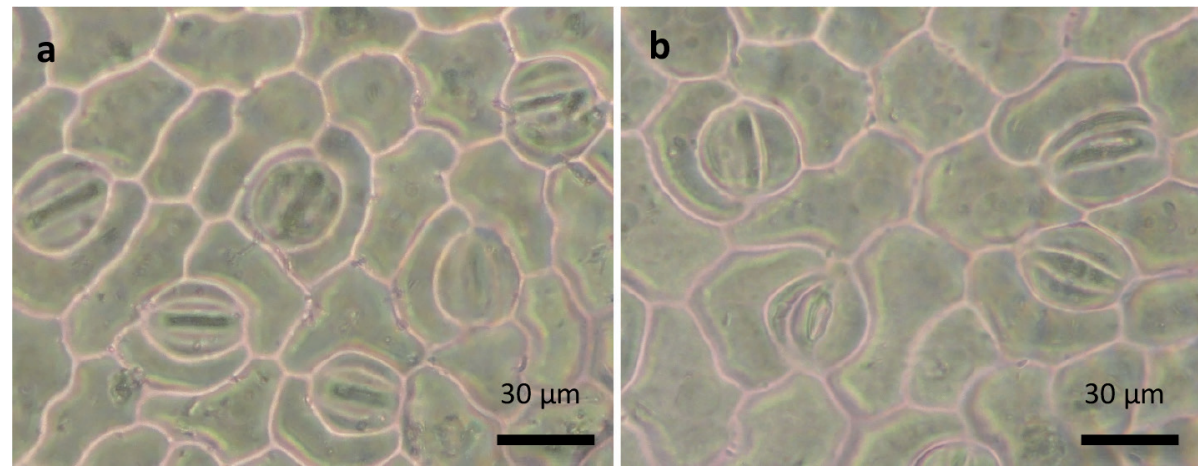

Figura 9. Densidad estomática en hojas del genotipo INIFAP P5 en diferentes condiciones hídricas. a) Densidad en hojas de plantas sin estrés y b) densidad en hojas con estrés. Chiapas, México. 2013-2014.

Figure 9. Stomatic density in leaves of genotype INIFAP P5 under different water conditions. a) Density in leaves of plant without water stress and b) density in leaves with water stress. Chiapas, Mexico. 2013-2014.

que presentó los mayores valores en cinco de las variables de estudio seleccionadas para determinar el ITS (Cuadro 3). Por el contrario, el genotipo INIFAP P4 se seleccionó como el más susceptible de acuerdo con la tasa de crecimiento relativo y el número de nudos totales, donde presentó los valores más bajos del ITS, aunque para el NHR fue uno de los más tolerantes, mientras que el INIFAP P7 fue uno de los más sensibles.
En relación con lo anterior, en la Figura 10 se muestra la apariencia del genotipo más tolerante y uno de los más susceptibles en cuanto a la TCR y NNT, en la cual se aprecia una diferencia notable entre las plantas que fueron sometidas a estrés y las que estuvieron a riego constante.

Al observar la correlación entre algunas de las variables de estudio, fue posible llevar a cabo el análisis de componentes principales $(\mathrm{CP})$ para las

Cuadro 3. Índice de tolerancia a sequía en plantas de cinco genotipos de C. canephora sometidos a dos condiciones hídricas (sin estrés y con estrés hídrico). Chiapas, México. 2013-2014.

Table 3. Index of drought tolerance in plant of five genotypes of $C$. canephora under to two water conditions (without and with water stress). Chiapas, Mexico. 2013-2014.

\begin{tabular}{lccccc}
\hline Genotipo & \multicolumn{5}{c}{ Variables } \\
\cline { 2 - 6 } & NHR & TCR & PRP & NNT & NHT \\
\hline INIFAP P7 & 64 & 280 & 280 & 233 & 684 \\
INIFAP P8 & 222 & 250 & 163 & 206 & 600 \\
INIFAP P9 & 273 & 240 & 175 & 264 & 418 \\
INIFAP P4 & 563 & 225 & 254 & 168 & 572 \\
INIFAP P5 & 605 & 440 & 375 & 654 & 5200 \\
\hline
\end{tabular}

NHR: número de hojas retenidas, TCR: tasa de crecimiento relativo, PRP: pares de ramas plagiotrópicas, NNT: número de nudos totales, NHT: número de hojas totales / NHR: Number of retained leaves, TCR: Relative growth rate, PRP: pairs of plagiotropic branches, NNT: Number of total nodes, NHT: number of total leaves. 


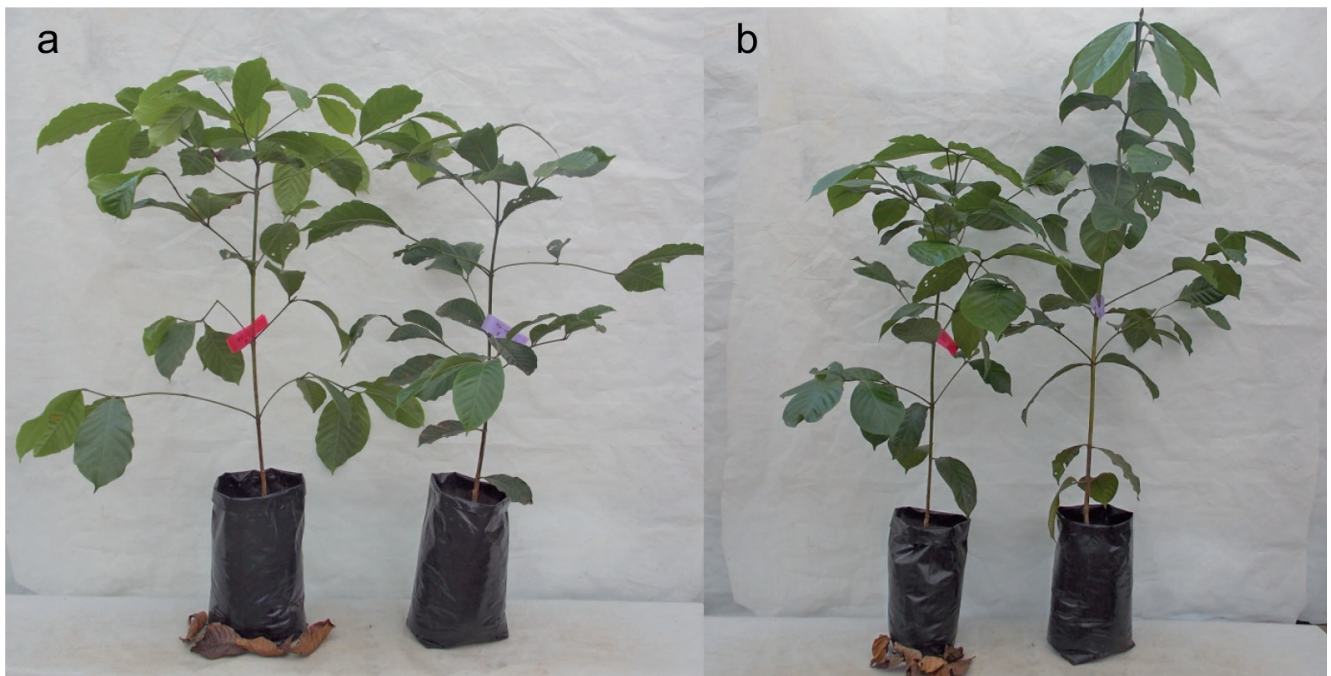

Figura 10. Aspecto de plantas de C. canephora a los sesenta días. a) INIFAP P5, plantas sometidas a estrés hídrico a punto de marchitez permanente (PMP) (izquierda) y a riego constante (derecha). b) INIFAP P4, plantas sometidas a estrés hídrico a punto de marchitez permanente (PMP) (izquierda) y a riego constante (derecha). Chiapas, México. 2013-2014.

Figure 10. Appearance of $C$. canephora plants at sixty days. a) INIFAP P5, plants subjected to water stress in permanent wilting point (PWP) (left) and constant irrigation (right). b) INIFAP P4, plants subjected to water stress in permanent wilting point (PWP) (left) and constant irrigation (right). Chiapas, Mexico. 2013-2014.

variables altura, diámetro de tallo, número de hojas totales, número de pares de ramas plagiotrópicas, nudos totales, hojas retenidas, hojas desprendidas, TCR y CRA. En dicho análisis el CP1 y CP2 explicaron el $62 \%$ de la variación acumulada.

El análisis discriminante permitió identificar que el genotipo INIFAP P5, tanto en la condición de CC como en PMP, presentó una misma agrupación, y se diferenció de forma significativa de las condiciones hídricas en la que se ubicaron el resto de los genotipos. Una respuesta similar fue observada para el genotipo INIFAP P9 y P4. Por el contrario, el genotipo INIFAP P7 en condición de PMP fue significativamente diferente que en la condición de CC. Estas diferencias confirman una mayor tolerancia del genotipo INIFAP P9 y mayor susceptibilidad del INIFAP P7.

Mientras, el análisis canónico permitió identificar a las variables número de hojas, hojas retenidas y número de hojas desprendidas, como las que más aportaron a las diferencias observadas.

Con base en los resultados de esta investigación, fue posible detectar que los genotipos presentaron diferentes mecanismos de tolerancia, tales como elongación de la raíz, mantenimiento de la TCR, acumulación de prolina y aumento de CRA. Lo cual repercute en el crecimiento de la planta, biomasa, retención de hojas y necrosamiento de las mismas. Debido a lo anterior, los genotipos presentaron diferente grado de tolerancia al déficit hídrico.

Fue posible detectar las variables de estudio asociadas con la tolerancia a sequía en cada genotipo; sin embargo, cabe destacar que el nivel de tolerancia a sequía entre los genotipos fue diferente de acuerdo con la variable de estudio, por lo que este puede cambiar de acuerdo con las variables seleccionadas.

Con base en el índice de tolerancia a sequía, el genotipo INIFAP P5 fue el más tolerante, mientras que el INIFAP P4 fue el más susceptible.

\section{LITERATURA CITADA}

AMECAFE (Asociación Mexicana de la Cadena Productiva del Café, A.C). 2012. Plan integral de promoción 
del café de México. http://amecafe.org.mx/backup/ pcm2012.pdf (consultado jun. 2015).

Avendaño, C.H., L.C. Trejo, C.C. López, G.J. Molina, V.A. Santacruz, y G.F. Castillo. 2005. Comparación de cuatro variedades de maíz (Zea mays L.) y su relación con la acumulación de prolina. Interciencia 30:560-564.

Barajas, J.E., A.J. Aldrete, H. Vargas, y U.J. López. 2004. La poda química en vivero incrementa la densidad de raíces en árboles jóvenes de Pinus greggii. Agrociencia 38:545-553.

Bates, L.S. 1973. Rapid determination of free proline for water-stress studies. Plant Soil 39:205-207.

Beadle, C.L. 1985. Plant growth analysis. In: J. Coombs et al., Techniques in bioproductivity and photosyntesis. $2^{\text {nd }}$ ed. Pergamon Press, GBR. p. 20-25.

Castañón, G., R. Cruz, R. Del Pino, E. Panzo, M. Montiel, y L. Filobello. 2000. Selección de líneas de maíz por resistencia a sequía. Agron. Mesoam. 11(1):163-169.

Castillo, M.I., M.R. Medina, I.E. González, y M.J. Pérez. 2008. Influencia del manejo del agua y del sustrato en la calidad de la planta de Eucalyptus grandis Hill ex maiden en contenedores. Rev. Forestal Baracoa 27(1):34-52.

Cheserek, J.J., and B.M. Gichimu. 2012. Drought and heat tolerance in coffee: a review. Int. Res. J. Agric. Sci. Soil Sci. 2:498-501.

DaMatta, F.M., and J.D. Ramalho. 2006. Impacts of drought and temperature stress on coffee physiology and production: a review. Braz. J. Plant. Physiol. 18:55-81.

De la Peña, I. 1977. El buen uso y manejo del agua de riego. Boletín técnico No. 8. Municipio de Sonora, Obregón, Sonora, MEX.

Frank, E., H. Eakin, and D. López-Carr. 2011. Social identity, perception and motivation in adaptation to climate risk in the coffee sector of Chiapas, México. Global Environ. Chang. 21:66-76.

González, L.M., L. Argentel, A. Estrada, N. Saldivar, y R. Ramírez. 2005. Evaluación de la tolerancia a la sequía en genotipos de trigo (Triticum aestivium L.) obtenidos en Cuba por inducción de mutaciones. Cultivos Trop. 26(3):65-69.

Guerrero, D.G. 2011. Captura de carbono en cafetales de la cuenca alta del río Pijijiapan, Chiapas. Tesis Lic., Universidad Nacional Autónoma de México, México, D.F., MEX

Kramer, P.J. 1974. Relaciones hídricas de suelos y plantas. Edutex S.A., MEX.

Lamz, A., y M.C. González. 2013. La salinidad como problema en la agricultura: la mejora vegetal una solución inmediata. Cultivos Trop. 34(4):31-42.
Llano, J.M., y L. Alcaraz. 2012. Análisis de pigmentos, peroxidasa, prolina y proteínas de tres especies de Paulownia bajo estrés hídrico. Rev. Mex. Cien. For. 3(9):69-80.

López-Peralta, M.C. 1984. Tolerance of tomato genotypes to osmotic stress in vitro. Ph.D. Thesis, Purdue University, USA.

Luna-Flores, W., H. Estrada-Medina, J.J. Jiménez-Osornio, y L.L. Pinzón-López. 2012. Efecto del estrés hídrico sobre el crecimiento y eficiencia del uso de agua en plántulas de tres especies arbóreas caducifolias. Terra Latinoam. 30:343-353.

Mamani-Rojas, P., y J. François-Ledent. 2014. Efecto de la sequía en la morfología, crecimiento y productividad de genotipos de papa (Solanum tuberosum L.) en Bolivia. Rev. Lat. Am. Papa 18(1):26-76.

Marraccini, P., F. Vinecky, G.S. Alves, H.J. Ramos, S. Elbelt, N.G. Vieira, F.A. Carneiro, P.S. Sujii, J.C. Alekcevetch, V.A. Silva, F.M. DaMatta, M.A. Ferrrao, T. Leroy, D. Pot, L.G. Vieira, F.R. Da Silva, and A.C. Andrade. 2012. Differentially expressed genes and proteins upon drought acclimation in tolerant and sensitive genotypes of Coffea canephora. J. Exp. Bot. 63:4191-4221.

Martínez, M.L., C.P. Moreno, y E. Rincón. 1994. Sobrevivencia y crecimiento de plántulas de un arbusto endémico de dunas costeras ante condiciones de sequía. Acta Bot. 26:53-62.

Mohamed, A.H., P.J. Harris, and J. Henderson. 2000. In vitro selection and characterization of a drought tolerant clone of Tagetes minuta. Plant Sci. 159:213-222.

Navarro-Sandoval, J., J. Vargas-Hernández, A. Gómez-Guerrero, L. Ruíz-Posadas, y P. Sánchez-García. 2013. Morfología, biomasa y contenido nutrimental en Abies religiosa con regímenes diferentes de fertilización en vivero. Agrociencia 47:707-721.

Paula, S. 2004. Análisis funcional de la capacidad de regeneración de tres especies de brezo rebrotadores (Erica scoparia, E. australis y E. arborea) tras perturbaciones severas recurrentes. Tesis Dr., Universidad de Cádiz, ESP.

Sáenz-Romero, C., G.E. Rehfeldt, N.L. Crookston, P. Duval, and J. Beaulieu. 2012. Spline models of contemporary, 2030, 2060 and 2090 climates for Michoacan state, México. Impacts on the vegetation. Rev. Fitot. Mex. 35:333-345.

Salas, J.A., M.E. Sanabria, y R. Pire. 2001. Variación en el índice y densidad estomática en plantas de tomate (Lycopersicon esculentum Mill.) sometidas a tratamientos salinos. Bioagro 12:99-104. 
Schroth, G., P. Laderach, J. Dempewolf, S. Philpott, J. Haggar, H. Eakin, T. Castillejos, J. García, L. Soto, R. Hernández, A. Eitzinger, and J. Ramírez-Villegas. 2009. Towards a climate change adaptation strategy for coffee communities and ecosystems in the Sierra Madre de Chiapas, México. Mitig. Adapt. Strateg. Glob. Change 14:605-625.

SHCP (Secretaría de Hacienda y Crédito Público). 2014. Panorama del café. http://www.financierarural.gob. $\mathrm{mx} /$ informacionsectorrural/Panoramas/Ficha $\% 20$ Caf\%C3\%A9.pdf (consultado mayo 2016).

SIAP (Servicio de Información Agroalimentaria y Pesquera). 2015. Convención internacional del café México 2015. http://www.gob.mx/siap/articulos/convencioninternacional-del-cafe-mexico-2015? idiom=es (consultado en mayo 2016).

Silva, E.M., P.C. Cavatte, L.E. Morais, E.F. Medina, and F.M. DaMatta. 2013. The functional divergence of biomass partitioning, carbon gain and water use in Coffea canephora in response to the water supply: implications for breeding aimed at improving drought tolerance. Environ. Exp. Bot. 87:49-57.

Toral, M., A. Manríquez, R. Navarro-Cerillo, D. Tersi, y P. Naulin. 2010. Características de los estomas, densidad e índice estomático en secuoya (Sequoia sempervirens) y su variación en diferentes plantaciones de Chile. Bosque 31:157-164.

Villar, R., J. Ruíz-Robleto, J.L. Quero, H. Poorter, F. Valladares, y T. Marañón. 2004. Tasas de crecimiento en especies leñosas: aspectos funcionales e implicaciones ecológicas. En: F. Valladares, editor, Ecología del bosque mediterráneo en un mundo cambiante. $2^{\text {a }}$ ed. Organismo Autónomo de Parques Nacionales, ESP. p. 191-227. 